\title{
Solving the Ginzburg-Landau equations by finite-element methods
}

\author{
Q. Du \\ Department of Mathematics, Michigan State University, East Lansing, Michigan 48224 \\ M. D. Gunzburger and J. S. Peterson \\ Department of Mathematics, Virginia Tech, Blacksburg, Virginia 24061
}

(Received 10 February 1992)

\begin{abstract}
We consider finite-element methods for the approximation of solutions of the Ginzburg-Landau equations of superconductivity. The methods are based on a discretization of the Euler-Lagrange equations resulting from the minimization of the free-energy functional. The discretization is effected by requiring the approximate solution to be a piecewise polynomial with respect to a grid. The magnetization versus magnetic field curves obtained through the finite-element methods agree well with analogous calculations obtained by other schemes. We demonstrate, both by analyzing the algorithms and through computational experiments, that finite-element methods can be very effective and efficient means for the computational simulation of superconductivity phenomena and therefore could be applied to determine macroscopic properties of inhomogeneous, anisotropic superconductors.
\end{abstract}

\section{INTRODUCTION}

The purpose of this paper is to demonstrate that finiteelement methods can be effectively used to determine the macroscopic properties of superconductors. The subject of our demonstration is homogeneous, isotropic superconductors described by the Ginzburg-Landau model. The efficiency and accuracy of these methods for these superconductors indicate that they may also be effectively used for studying the physical behavior of inhomogeneous, anisotropic superconductors and for designing devices using such materials.

Suppose that one wishes to effect a numerical simulation of electromagnetic phenomena in a sample of superconducting material which is part of some device. For a type-I superconductor, or for a type-II superconductor in the presence of external fields smaller than $H_{c 1}$, this poses little problem since, except for possible edge effects, all variables to be approximated are smooth with respect to the scale of the sample size. Finite-element methods for just this situation are considered in Du, Gunzburger, and Peterson. ${ }^{1}$ However, a sample of a type-II superconductor in the presence of external fields between $H_{c 1}$ and $H_{c 2}$ is impossible to simulate, on currently available computers, because of the appearance of vortexlike structures whose spacing is very small compared with the sample size. For example, if one were to use a finite-element or finite-difference method, then the number of degrees of freedom necessary to well approximate such phenomena would be prohibitively large.

The inability to perform simulations for typical material samples of type-II superconductors gives rise to the use of the common practice of neglecting the effects due to sample boundaries. Thus one assumes that one is far removed from the boundary of the superconducting sample and that in such regions the physically relevant variables, e.g., the magnetic field, the current, etc., are, in some sense, periodic. The use of a periodic model allows one to focus on a piece of the sample that is of roughly the same size as that of the scale of variations in the interesting phenomena. In this case it is easily possible to resolve these phenomena on currently available computers. In fact, the results given in this paper were obtained on a Macintosh II.

Periodic Ginzburg-Landau models have been used in the past as a setting for analyzing and approximating phenomena in type-II superconductors. ${ }^{2-9}$ Most of these deal with some sort of series solution. One notable exception is that of Doria, Gubernatis, and Rainer, ${ }^{4}$ which uses a Monte Carlo-simulated annealing approach and represents the best-known effort so far for simulating type-II superconductors. The particular periodic Ginzburg-Landau model considered here is studied in detail in Du, Gunzburger, and Peterson ${ }^{10}$ and is closely related to the models used in the other references cited. We note that there are some discrepancies and inconsistencies with the various specific models employed in the literature; in Du, Gunzburger, and Peterson, ${ }^{10}$ many of these are reconciled or at least explained.

Finite-element methods are characterized by approximate solutions that are piecewise polynomial functions with respect to some grid. A specific finite-element scheme is defined by choosing a particular space of such functions in which to seek approximate solutions. Then the discrete equations are deduced by requiring that in some sense the residual obtained by substituting the approximate solution into the governing equations is orthogonal to all functions belonging to that space.

Finite-element methods have proven very effective in a variety of other applications, e.g., solid mechanics, fluid mechanics, electromagnetics, heat transfer, semiconductors, etc. There are also many fine books about finiteelement methods; here, we merely cite four "classics."11-14 Moreover, in many settings, a rigorous 
mathematical analyses of the convergence of approximations and estimates for the errors induced by finiteelement discretizations have been carried out. However, to our knowledge, finite-element methods have not been heretofore applied to Ginzburg-Landau models of superconductivity. We have succeeded in developing, analyzing, and implementing finite-element methods for superconductivity applications. First, in Du, Gunzburger, and Peterson, ${ }^{1}$ we developed and analyzed finite-element algorithms for superconductors having mixed states only near boundaries, e.g., type-I superconductors. Then, in Du, Gunzburger, and Peterson, ${ }^{15}$ we treated finite-element methods for periodic superconductivity models that are applicable to type-II superconductors. Here our main goal is to present the results of some computational experiments for the second, more interesting, setting.

The plan of the rest of the paper is as follows. In the next section we describe the periodic Ginzburg-Landau model. In Sec. III we describe the use of finite-element algorithms for the approximation of solutions of the equations of Sec. II. In the last section, we illustrate the effectiveness of our algorithms by presenting the results of some computational experiments.

\section{PERIODIC MODEL}

Throughout, we only deal with two-dimensional Euclidean space. Given any two noncolinear vectors $\mathbf{t}_{1}$ and $\mathbf{t}_{2}$, we say that a function $f(\mathbf{x})$ is periodic with respect to the lattice determined by $\mathrm{t}_{1}$ and $\mathrm{t}_{2}$ if

$$
f\left(\mathbf{x}+\mathbf{t}_{k}\right)=f(\mathbf{x}) \text { for } k=1,2 \text { and for all points } \mathbf{x} .
$$

Here $f$ may be scalar or vector valued and may be real or complex valued. The vectors $\mathrm{t}_{k}, k=1,2$, will be referred to as lattice vectors; without loss of generality, we assume that the counterclockwise angle between $t_{1}$ and $t_{2}$ is less than $\pi$. For the sake of brevity, we will often refer to functions satisfying (1) merely as being periodic. We will not need to specify explicitly the lattice vectors $t_{1}$ and $\mathbf{t}_{2}$ until we consider, in Sec. IV, computational examples.

Given any point $\mathbf{P}$ a cell of the lattice with respect to the point $\mathbf{P}$ is the parallelogram $\Omega_{P}$ depicted in Fig. 1. The boundary of the cell $\Omega_{P}$ is denoted by $\Gamma_{P}$. When $\mathbf{P}$ corresponds to the origin, we denote the corresponding cell by $\Omega$ and its boundary by $\Gamma$. We denote by $|\Omega|$ the area of the cell $\Omega_{p}$; note that $|\Omega|$ is independent of the choice for P.

The variables employed in Ginzburg-Landau models for superconductivity are the real, vector-valued magnetic potential $\mathbf{A}$ and the complex, scalar-valued order parameter $\psi$. From these one may deduce (appropriately nondimensionalized) physical variables, e.g., the magnetic field $H=\nabla \times \mathbf{A}$ and the density of superconducting charge carriers $N_{s}=|\psi|^{2}$. Two pairs $(\psi, \mathbf{A})$ and $(\widetilde{\psi}, \widetilde{\mathbf{A}})$ are said to gauge equivalent if, for some $\chi$,

$$
\widetilde{\psi}=\psi e^{i \chi} \text { and } \widetilde{\mathbf{A}}=\mathbf{A}+\frac{1}{\kappa} \nabla \chi .
$$

Periodicity assumptions about the physical variables,

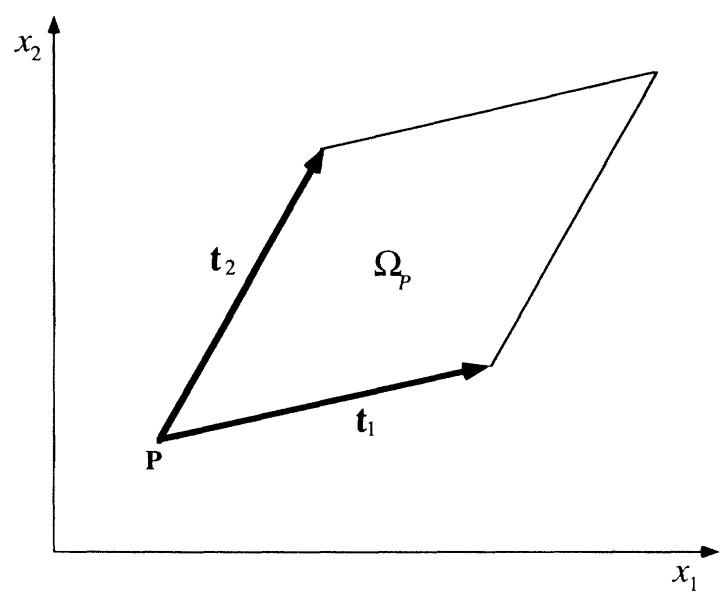

FIG. 1. Cell $\Omega_{P}$ determined by the lattice vectors $t_{1}$ and $t_{2}$ and the point $\mathbf{P}$.

i.e., $H, N_{s}$, and the Gibbs free energy, can be expressed in terms of the magnetic potential $\mathbf{A}$ and order parameter $\psi$ as

$$
\boldsymbol{\nabla} \times \mathbf{A},|\psi|, \text { and }\left[\frac{1}{\kappa} \boldsymbol{\nabla} \phi-\mathbf{A}\right] \text { are periodic, }
$$

where $\phi$ denotes the phase of the order parameter, i.e., $\psi=|\psi| e^{i \phi}$, and $\kappa$ is the Ginzburg-Landau parameter. Recall that, here and throughout, periodicity is defined with respect to the lattice determined by the vectors $t_{1}$ and $t_{2}$.

The average magnetic field $\bar{B}$ over a cell $\Omega_{P}$ is defined by

$$
\bar{B}=\frac{1}{|\Omega|} \int_{\Omega_{P}} H d \Omega=\frac{1}{|\Omega|} \int_{\Omega_{P}} \nabla \times \mathbf{A} d \Omega .
$$

Note that $\bar{B}$ is independent of the choice of cell, i.e., of the choice of the point $P$. The relation

$$
\kappa \bar{B}|\Omega|=2 \pi n,
$$

between the average magnetic field and the area of a lattice cell, results from the "fluxoid quantization condition;" here, the positive integer $n$ is the number of fluxoids associated with a lattice cell.

Let

$$
\mathbf{A}_{0}=\frac{\bar{B}}{2}\left[\begin{array}{c}
x_{2} \\
-x_{1}
\end{array}\right)
$$

and

$$
g_{k}(\mathbf{x})=-\frac{\bar{B}}{2}\left(\mathbf{x} \times \mathbf{t}_{k}\right), \quad k=1,2 .
$$

If $\mathbf{A}$ and $\psi=|\psi| e^{i \phi}$ satisfy (2), it is shown in Du, Gunzburger, and Peterson ${ }^{10}$ that $(\psi, \mathbf{A})$ is gauge equivalent to $\left(\zeta, \mathbf{Q}-\mathbf{A}_{0}\right)$, where

$$
|\zeta| \text { and } \mathbf{Q} \text { are periodic , }
$$

$$
\boldsymbol{\nabla} \cdot \mathbf{Q}=\mathbf{0},
$$

and 


$$
\omega\left(\mathbf{x}+\mathbf{t}_{k}\right)-\omega(\mathbf{x})=\kappa g_{k}(\mathbf{x}), \quad k=1,2,
$$

where $\omega$ denotes the phase of $\zeta$, i.e., $\zeta=|\zeta| e^{i \omega}$. Furthermore, the magnetic field $H$ and the density of superconducting charge carriers may be recovered from $\zeta$ and $\mathbf{Q}$ through the relations

$$
H=\nabla \times \mathbf{A}=\nabla \times \mathbf{Q}+\bar{B} \text { and } N_{s}=|\psi|^{2}=|\zeta|^{2} .
$$

The Ginzburg-Landau equations are a consequence of the basic postulate of the Ginzburg-Landau theory, namely, that a superconducting material is in a state such that the Gibbs free energy is minimized. In terms of an order parameter $\zeta$ and a reduced magnetic potential $\mathbf{Q}$ which satisfy (5)-(7), the Ginzburg-Landau equations, posed over a lattice cell $\Omega_{P}$, are given by ${ }^{10}$

$$
\begin{aligned}
\left(\frac{i}{\kappa} \boldsymbol{\nabla}-\mathbf{A}_{0}\right) \cdot\left(\frac{i}{\kappa} \nabla-\mathbf{A}_{0}\right) \zeta+\left(|\mathbf{Q}|^{2}+|\zeta|^{2}-1\right) \zeta \\
+2 \mathbf{Q} \cdot\left(\frac{i}{\kappa} \boldsymbol{\nabla}-\mathbf{A}_{0}\right) \zeta=0 \text { in } \Omega_{P}
\end{aligned}
$$

and

$\boldsymbol{\nabla} \times \boldsymbol{\nabla} \times \mathbf{Q}+|\zeta|^{2} \mathbf{Q}+\operatorname{Re}\left\{\zeta^{*}\left[\frac{i}{\kappa} \boldsymbol{\nabla}-\mathbf{A}_{0}\right] \zeta\right\}=0$ in $\Omega_{P}$

where $\operatorname{Re}$ denotes the real part. Of course, $\mathbf{Q}$ satisfies (6)

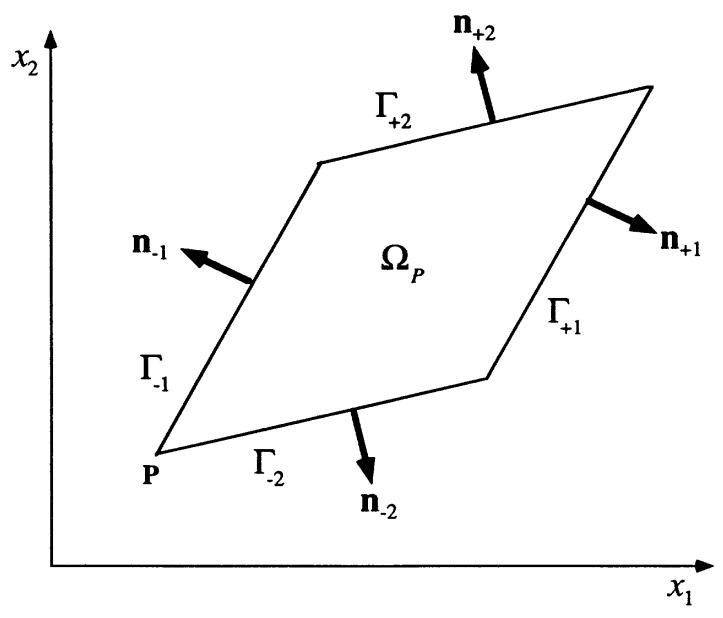

FIG. 2. Boundary segments and normal vectors of the lattice cell $\Omega_{P}$.

as well.

Denote the four sides of the parallelogram $\Omega_{P}$ by $\Gamma_{+1}$, $\Gamma_{-1}, \Gamma_{+2}$, and $\Gamma_{-2}$, using the convention of Fig. 2 . Note that, for $k=1$ or $2, \Gamma_{+k}$ is the locus of points $y$ such that $\mathbf{y}=\mathbf{x}+\mathbf{t}_{k}$ for $\mathbf{x} \in \Gamma_{-k}$.

The "periodicity" conditions (5) and (7) on $\zeta$ and $Q$ over all of space induce the following conditions with respect to a lattice cell:

$$
\begin{aligned}
& \zeta\left(\mathbf{x}+\mathbf{t}_{k}\right)=\zeta(\mathbf{x}) e^{i \kappa g_{k}(\mathbf{x})} \text { for all } \mathbf{x} \text { on } \Gamma_{-k}, \quad k=1,2, \\
& \mathbf{Q}\left(\mathbf{x}+\mathbf{t}_{k}\right)=\mathbf{Q}(\mathbf{x}) \text { for all } \mathbf{x} \text { on } \Gamma_{-k}, \quad k=1,2, \\
& \left.e^{-i \kappa g_{k}(\mathbf{x})}\left[\left(\frac{i}{\kappa} \nabla-\mathbf{A}_{0}\right] \zeta+\mathbf{Q} \zeta\right]\right|_{\mathbf{x}+\mathbf{t}_{k}} \cdot \mathbf{n}_{+k}+\left.\left[\left(\frac{i}{\kappa} \nabla-\mathbf{A}_{0}\right] \zeta+\mathbf{Q} \zeta\right]\right|_{\mathbf{x}} \cdot \mathbf{n}_{-k}=0 \text { for all } \mathbf{x} \text { on } \Gamma_{-k}, k=1,2,
\end{aligned}
$$

and

$$
\boldsymbol{\nabla} \times\left.\mathbf{Q}\right|_{\mathbf{x}+\mathbf{t}_{k}}=\boldsymbol{\nabla} \times\left.\mathbf{Q}\right|_{\mathbf{x}} \text { for all } \mathbf{x} \text { on } \Gamma_{-k}, k=1,2 .
$$

The periodic Ginzburg-Landau model we employ is thus defined by (9)-(14), along with (6). Note that, as a result of (10), the current $J=\nabla \times \nabla \times Q$ is given by

$$
\mathbf{J}=-|\zeta|^{2} \mathbf{Q}-\operatorname{Re}\left\{\zeta^{*}\left[\frac{i}{\kappa} \nabla-\mathbf{A}_{0}\right] \zeta\right\}
$$

\section{FINITE-ELEMENT METHODS}

Intermediate between the minimization principle and the Ginzburg-Landau equations (9)-(14) is a weak form of these equations. It is the latter that forms the basis for defining finite-element discretizations. One can go from the weak form to the strong form (9)-(14) through integration by parts procedures. Here we will merely provide the necessary relations in a discrete context; details may be found in, e.g., Du, Gunzburger, and Peterson. ${ }^{10}$

We will seek an approximate order parameter $\xi^{h}$ and reduced magnetic potential $\mathbf{Q}^{h}$ such that the discrete GinzburgLandau equations 


$$
\begin{aligned}
\int_{\Omega_{P}} \operatorname{Re}\{ & \left.\left\{\frac{i}{\kappa} \boldsymbol{\nabla}-\mathbf{A}_{0}\right) \xi^{h \cdot}\left(-\frac{i}{\kappa} \boldsymbol{\nabla}-\mathbf{A}_{0}\right) \widetilde{\zeta}^{h *}\right\} d \Omega \\
& +\int_{\Omega_{P}} \operatorname{Re}\left\{\widetilde{\xi}^{h *}\left(\frac{i}{\kappa} \boldsymbol{\nabla}-\mathbf{A}_{0}\right) \xi^{h}+\xi^{h}\left(-\frac{i}{\kappa} \boldsymbol{\nabla}-\mathbf{A}_{0}\right) \xi^{h *}\right\} \cdot \mathbf{Q}^{h} d \Omega+\int_{\Omega_{P}}\left(\left|\mathbf{Q}^{h}\right|^{2}+\left|\xi^{h}\right|^{2}-1\right) \operatorname{Re}\left\{\xi^{h} \widetilde{\xi}^{h *}\right\} d \Omega=0
\end{aligned}
$$

and

$$
\int_{\Omega_{P}}\left(\boldsymbol{\nabla} \times \mathbf{Q}^{h} \cdot \boldsymbol{\nabla} \times \widetilde{\mathbf{Q}}^{h}+\boldsymbol{\nabla} \cdot \mathbf{Q}^{h} \cdot \boldsymbol{\nabla} \cdot \widetilde{\mathbf{Q}}^{h}+\left|\xi^{h}\right|^{2} \mathbf{Q}^{h} \cdot \widetilde{\mathbf{Q}}^{h}+\operatorname{Re}\left\{\xi^{h *}\left(\frac{i}{\kappa} \boldsymbol{\nabla}-\mathbf{A}_{0}\right) \xi^{h}\right\} \cdot \widetilde{\mathbf{Q}}^{h}\right) d \Omega=0
$$

hold for suitably chosen test functions $\widetilde{\zeta}^{h}$ and $\widetilde{\mathbf{Q}}^{h}$. All that remains is to indicate what type of functions are to be used for the approximate solution $\left(\xi^{h}, Q^{h}\right)$ and the test functions $\widetilde{\zeta}^{h}$ and $\widetilde{Q}^{h}$. Here we use finite-element methodology to choose these various functions.

The starting point for defining a finite-element method for a particular problem such as (16) and (17) is to subdivide the lattice cell $\Omega_{P}$ into smaller subregions, called elements, which are usually chosen to be quadrilaterals or triangles. Here we will employ the latter. For example, given any two positive integers $N_{1}$ and $N_{2}$, one may subdivide the parallelogram $\Omega_{P}$ into $2 N_{1} N_{2}$ triangles in the manner depicted in Fig. 3. The triangles in the subdivision are denoted by $\Omega_{j}, j=1, \ldots, n_{t}=2 N_{1} N_{2}$.

The second ingredient in the finite-element recipe is to define a space of piecewise polynomial functions with respect to the subdivision of $\Omega_{P}$. The two most practical choices are continuous piecewise linear and piecewise quadratic polynomials. Here we only consider the latter. Given a subdivision of $\Omega_{P}$ into the triangles $\Omega_{j}$, $j=1, \ldots, n_{t}$, a continuous piecewise quadratic polynomial is a function that is a quadratic polynomial in $x_{1}$ and $x_{2}$ in each triangle $\Omega_{j}$ and is continuous on $\bar{\Omega}_{P}$. It is easy to show that such a function is completely determined by its values at the vertices and midsides of the tri-

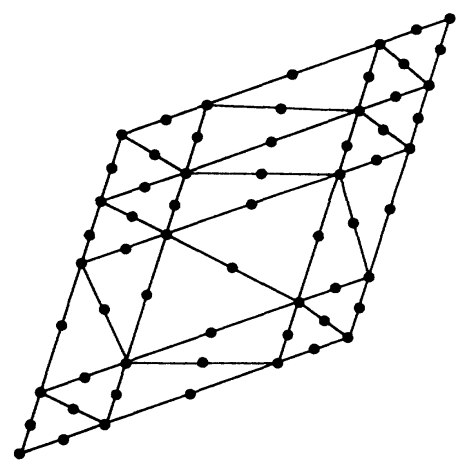

FIG. 3. Subdivision of $\Omega_{P}$ into $2 N_{1} N_{2}=24$ triangles; $N_{1}=3$, $N_{2}=4$. The $\left(2 N_{1}+1\right)\left(2 N_{2}+1\right)=63$ nodes are indicated by the solid circles. angles $\Omega_{j}$. These points are the nodes associated with the finite-element functions used. For the example triangulation depicted in Fig. 3, the $n_{n}=\left(2 N_{1}+1\right)\left(2 N_{2}+1\right)$ nodes are depicted in that figure. We will denote nodes associated with the piecewise polynomial functions by $\mathbf{z}_{s}$, $s=1, \ldots, n_{n}$.

The next ingredient, which is crucial to the efficiency of finite-element methods, is to choose a set of basis functions that have as small support as possible, i.e., that are nonvanishing over a small a subset of $\Omega_{P}$ as possible. Such basis functions are easily defined as follows. For $s=1, \ldots, n_{n}$, let $\Phi_{x}(\mathbf{x})$ be the unique function which is a quadratic polynomial in each triangle $\Omega_{j}, j=1, \ldots, n_{t}$, which is continuous over $\bar{\Omega}_{P}$ and which satisfies $\Phi_{s}\left(z_{l}\right)=\delta_{s l}$. Thus $\Phi_{s}(\mathbf{x})$ is unity at the node $z_{s}$ and vanishes at all the other nodes. The support of $\Phi_{k}(\mathbf{x})$ is simply those triangles for which the node $z_{s}$ is either a midside or a vertex. In an arrangement such as the one depicted in Fig. 3, the support of any of the basis functions $\Phi_{s}(\mathbf{x})$ extends over at most six triangles regardless of how large one chooses $N_{1}$ and $N_{2}$.

We seek approximations $\xi^{h}$ to the order parameter $\zeta$ and $\mathbf{Q}^{h}$ to the reduced magnetic potential $\mathbf{Q}$ such that the real and imaginary parts of $\xi^{h}$ and the components of $\mathbf{Q}^{h}$ are continuous piecewise quadratic polynomials. Any such function can be expressed as a linear combination of the basis functions $\Phi_{s}(\mathbf{x}), s=1, \ldots, n_{n}$. Thus, for some complex constants $\xi_{s}$ and constant vectors $\mathbf{Q}_{s}$, $s=1, \ldots, n_{n}$, we have that

$\xi^{h}(\mathbf{x})=\sum_{s=1}^{n_{n}} \zeta_{s} \Phi_{s}(\mathbf{x})$ and $\mathbf{Q}^{h}(\mathbf{x})=\sum_{s=1}^{n_{n}} \mathbf{Q}_{s} \Phi_{s}(\mathbf{x})$

Note that $\zeta_{s}$ and $\mathbf{Q}_{s}$ are simply the nodal values of $\zeta^{h}$ and $\mathbf{Q}^{h}$, respectively, i.e., $\xi^{h}\left(\mathbf{z}_{s}\right)=\xi_{s}$ and $\mathbf{Q}^{h}\left(\mathbf{z}_{s}\right)=\mathbf{Q}_{s}$, $s=1, \ldots, n_{n}$.

The discrete equations (16) and (17) do not account for all the "periodicity" conditions (11)-(14). Fortunately, the derivative conditions (13) and (14) are natural to the weak formulation on which (16) and (17) are based; (see 
Du, Gunzburger, and Peterson $\left.{ }^{10}\right)$. Consequently, these are accounted for by (16) and (17). However, (11) and (12) are essential conditions with respect to the weak formulation and must be explicitly accounted for. To this end we require that

$$
\begin{aligned}
& \xi^{h}\left(\mathbf{z}_{s}+\mathbf{t}_{k}\right)=\xi^{h}\left(\mathbf{z}_{s}\right) e^{i \kappa g_{k}\left(\mathbf{z}_{s}\right)} \\
& \text { for all } z_{s} \text { on } \Gamma_{-k}, k=1,2 \text {, }
\end{aligned}
$$

and

$\mathbf{Q}\left(\mathbf{z}_{s}+\mathbf{t}_{k}\right)=\mathbf{Q}\left(\mathbf{z}_{s}\right)$ for all $\mathbf{z}_{s}$ on $\Gamma_{-k}, \quad k=1,2$.

Note that the periodicity conditions (11) and (12) are enforced only at the nodes on the boundary. One easily sees that (20) implies that $\mathbf{Q}^{h}\left(\mathbf{x}+\mathbf{t}_{k}\right)=\mathbf{Q}^{h}(\mathbf{x})$ for all points $\mathbf{x}$ on $\Gamma_{-k}, k=1,2$. However, in general, $\zeta^{h}\left(\mathbf{x}+\mathbf{t}_{k}\right)$ $\neq \xi^{h}(\mathbf{x}) e^{i \kappa g_{k}(x)}$ if a boundary point $\mathrm{x}$ is not a node. [Because of the multiplicative term $e^{i \kappa g_{k}(x)}$, it is impossible for the polynomial functions $\xi^{h}$ to satisfy (11) exactly.]

All that remains to define completely the discrete equations is to choose the test functions $\xi^{h}$ and $Q^{h}$ in (16) and (17). These are chosen in a symmetric manner; i.e., they are also taken to be piecewise quadratic polynomial functions that satisfy the essential constraints (19) and (20).

Here we do not delve into the details of the implementation of the method described above, except to say that there are no great difficulties over and above those encountered when applying finite-element methods in other settings. However, we comment on the accuracy and complexity of the approximations obtained in this manner. In the first place it can be rigorously ${ }^{15}$ shown that in a root-mean-square sense, i.e., measured in $L^{2}\left(\Omega_{P}\right)$ norms, the differences $\left(\zeta-\xi^{h}\right)$ and $\left(\mathbf{Q}-\mathbf{Q}^{h}\right)$ are of order $h^{3}$ and the differences $\nabla\left(\zeta-\xi^{h}\right)$ and $\nabla\left(Q-Q^{h}\right)$ are of order $h^{2}$, where $h$ denotes the largest diameter of any of the triangles in the subdivision. For a triangulation such as the one depicted in Fig. 3, the number of real discrete nonlinear equations is roughly 4 times the number of nodes since there are four unknowns at each node, i.e., the real and imaginary parts of $\xi^{h}$ and the components of $\mathbf{Q}^{h}$.

The input data for the finite-element method consist partly of the parameters that uniquely specify the periodic Ginzburg-Landau model. These are given in the following list.

(i) The Ginzburg-Landau parameter $\kappa$. Since we are presuming the existence of a vortex lattice structure, we must have that $\kappa>1 / \sqrt{2}$.

(ii) The positive integer $n$, the number of fluxoids associated with a lattice cell.

(iii) The position of the point $\mathbf{P}$, which determines the position of the lattice cell with respect to the origin; the obvious and usual choice for $\mathbf{P}$ is the origin itself.

(iv) The average magnetic field $\bar{B}$; for a given value of $\kappa, \bar{B}$ may be chosen such that $0<\bar{B}<\kappa$. (v) The directions and relative magnitudes of the lattice vectors $t_{1}$ and $t_{2}$. Without loss of generality, one may choose $t_{1}$ to be aligned with the $x_{1}$ axis. One also chooses $\vartheta \neq 0$, the angle between $t_{1}$ and $t_{2}$, and $\gamma>0$, the ratio of the magnitudes of $t_{2}$ and $t_{1}$. The absolute magnitudes of $t_{1}$ and $t_{2}$ are then determined with the help of the fluxoid quantization condition, i.e., $|\Omega|=2 \pi n / \kappa \bar{B}$.

Thus the parameters that determine the periodic model are $\mathbf{P}, \kappa, n, \gamma, \vartheta$, and $\bar{B}$. The only other input datum required by the finite-element method is geometrical information about the grid, e.g., the positions of the nodes.

The reduced magnetic potential $\mathbf{Q}$ and order parameter $\zeta$ are the primary dependent variables employed in the periodic Ginzburg-Landau model. However, of more interest as output data are the magnetic field $H$, the current $\mathrm{J}$, and the density of superconducting charge carriers $N_{s}$. These may be determined from $\zeta$ and $\mathbf{Q}$ through relations (8) and (15).

For specified values for the input parameters, one would like to know the corresponding external magnetic field $H_{e}$. the best method for determining $H_{e}$ is developed in Doria, Gubernatis, and Rainer, ${ }^{16}$ where it is shown that

$$
\begin{aligned}
& H_{e}=\left.\frac{1}{\bar{B}|\Omega|} \int_{\Omega_{P}}\left(\frac{1}{2}|| \frac{i}{\kappa} \nabla+\mathbf{Q}-\mathbf{A}_{0}\right) \zeta\right|^{2} \\
& \left.+\mid \nabla \times Q+\bar{B})\left.\right|^{2}\right] d \Omega .
\end{aligned}
$$

Thus, once $(\zeta, Q)$ has been obtained from a given set of input parameters, $H_{e}$ can be determined by evaluating integrals. Another quantity of interest is the magnetization (or magnetic moment per unit volume) $M$ defined by

$$
-4 \pi M=H_{e}-\bar{B}
$$

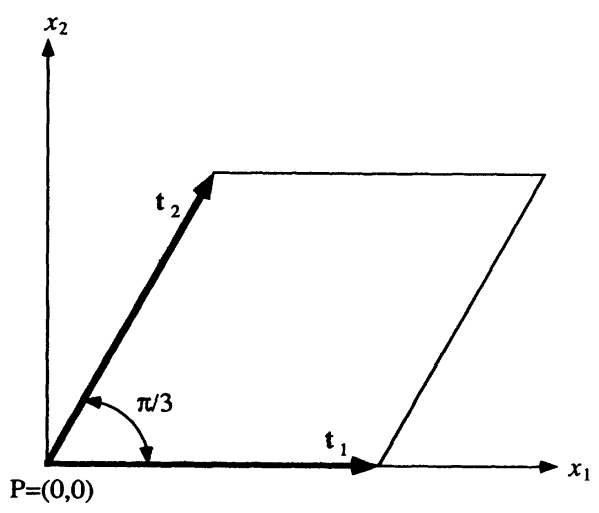

FIG. 4. Lattice cell $\Omega$ for an equilateral triangular lattice. 
If the piecewise quadratic finite-element approximations $\zeta^{h}$ and $\mathbf{Q}^{h}$ are substituted into (8), (15), (21), and (22), then one can deduce ${ }^{15}$ that the approximations to the magnetic field, current, external field, and magnetization so obtained are $O\left(h^{2}\right)$ and that for the density of superconducting charge carriers is $O\left(h^{3}\right)$.

\section{COMPUTATIONAL RESULTS}

We consider the most interesting periodicity structure, namely, that corresponding to an equilateral triangular lattice having one fluxoid associated with each lattice cell. It is well known that such a lattice yields the smallest value for the Gibbs free energy. For this case we have
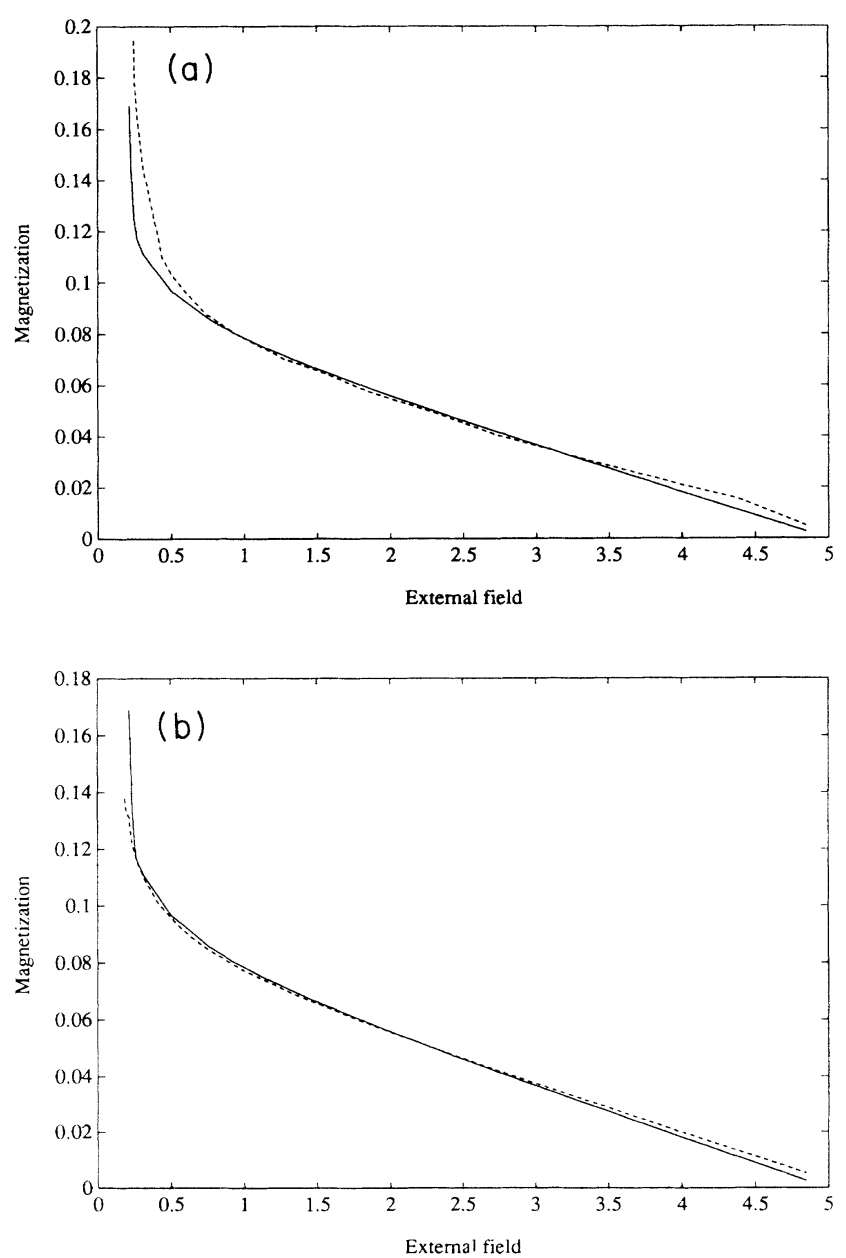

FIG. 5. (a) Magnetization ( $-4 \pi M)$ vs external field $\left(H_{e}\right)$ for $\kappa=5$ : finite elements with $N_{1}=N_{2}=3$ (solid curve) and Monte Carlo-simulated annealing ${ }^{4}$ (dashed curve) (Ref. 4). (b) Magnetization $(-4 \pi M)$ vs external field $\left(H_{e}\right)$ for two different grids and for $\kappa=5: \quad N_{1}=N_{2}=3$ (solid curve) and $N_{1}=N_{2}=4$ (dashed curve).
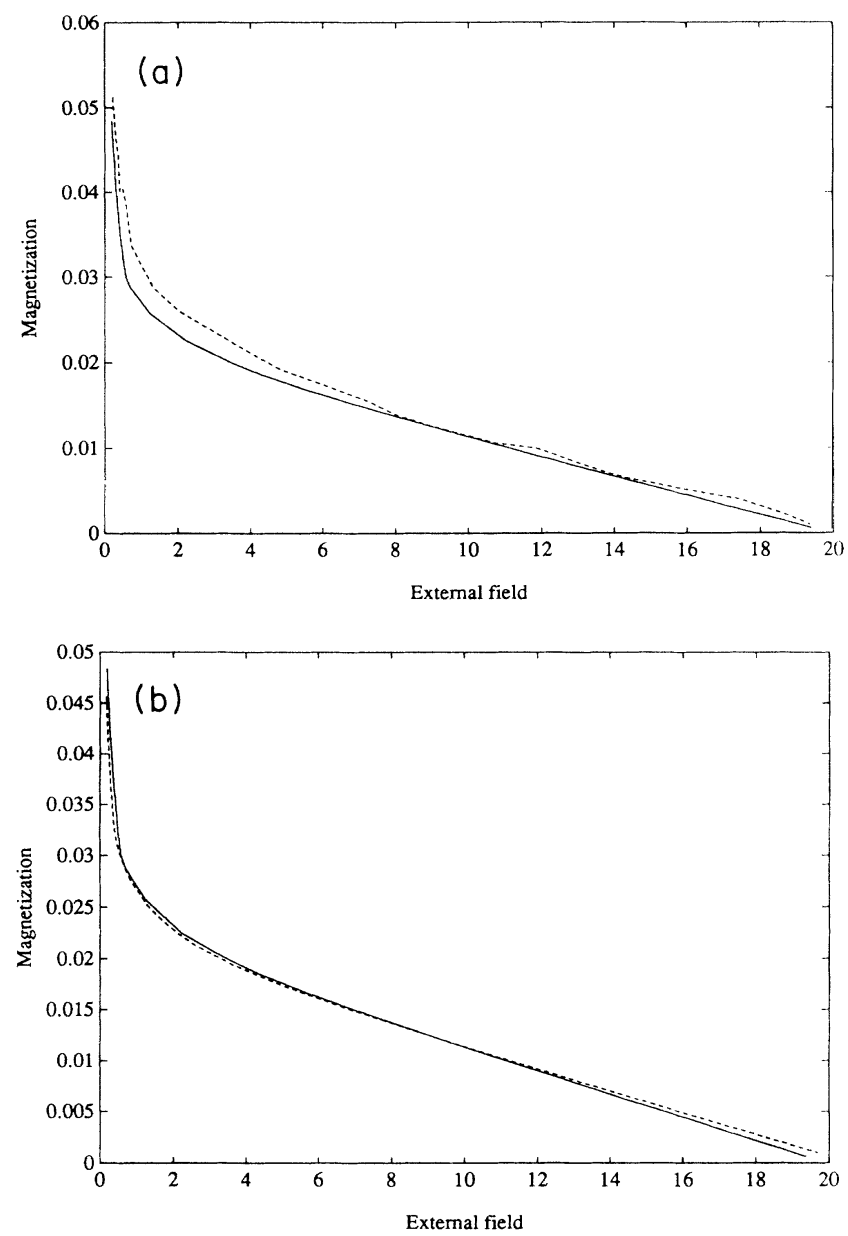

FIG. 6. (a) Magnetization $(-4 \pi M)$ vs external field $\left(H_{e}\right)$ for $\kappa=20$ : finite elements with $N_{1}=N_{2}=3$ (solid curve) and Monte Carlo-simulated annealing ${ }^{4}$ (dashed curve) (Ref. 4). (b) Magnetization $(-4 \pi M)$ vs external field $\left(H_{e}\right)$ for two different grids and for $\kappa=20: N_{1}=N_{2}=3$ (solid curve) and $N_{1}=N_{2}=4$ (dashed curve).

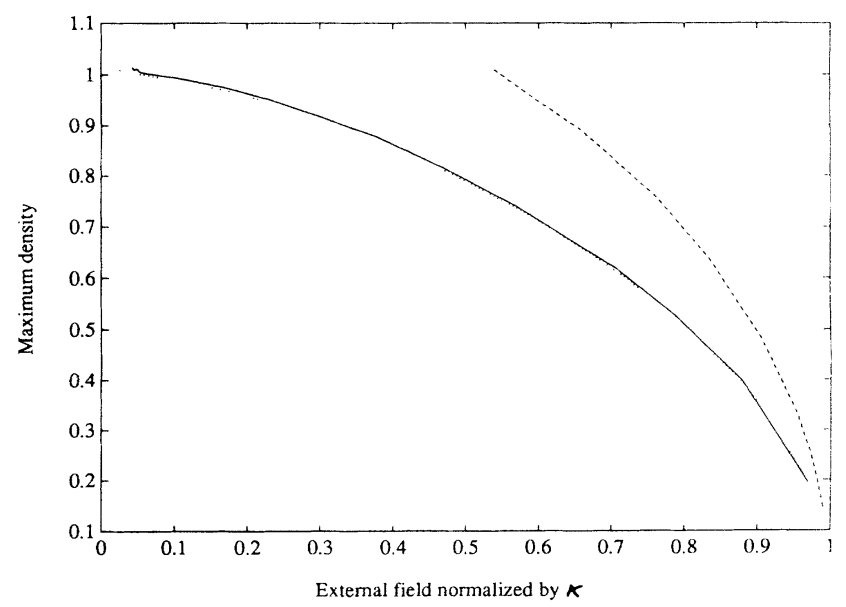

FIG. 7. Maximum value of the density of superconducting charge carriers $\left(N_{s}\right)$ vs the ratio of the external field to the Ginzburg-Landau parameter $\left(H_{e} / \kappa\right): \kappa=20$ (dotted curve), $\kappa=5$ (solid curve), and $\kappa=1$ (dashed curve). 
that $n=1, \gamma=1$, and $\vartheta=\pi / 3$. Then the area $|\Omega|$ of any lattice cell is given by $|\Omega|=\sqrt{3}\left|t_{1}\right|^{2} / 2=2 \pi / \kappa \bar{B}$, so that

$$
\mathbf{t}_{1}=\left(\frac{4 \pi}{\sqrt{3} \kappa \bar{B}}\right)^{1 / 2} \mathbf{i}_{1}
$$

and

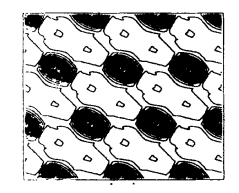

(a)

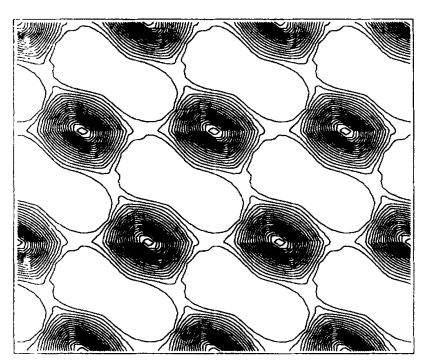

(b)

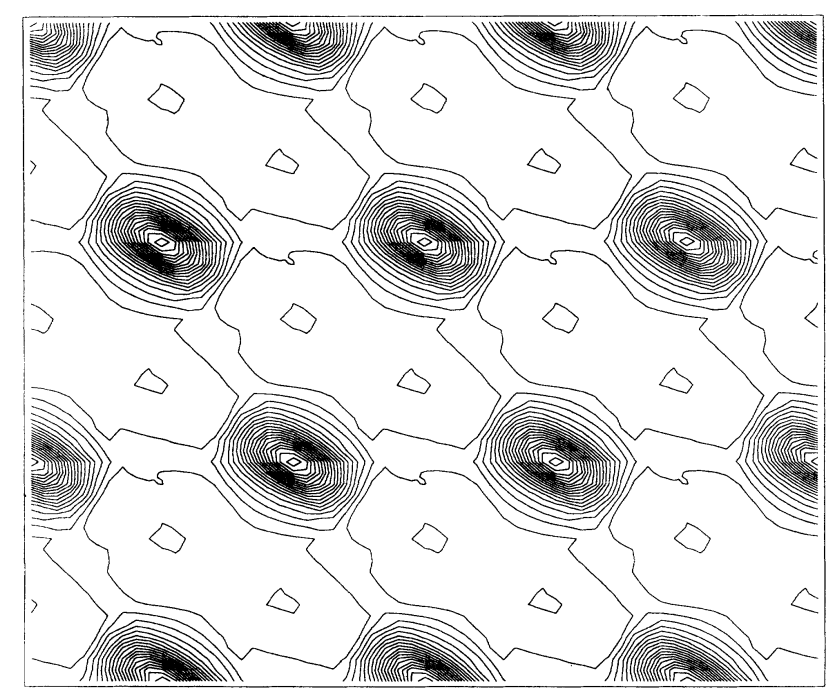

(c)

FIG. 8. (a) Level curves of $N_{s}$, the density of superconducting charge carriers, for $\kappa=20, \bar{B}=2 \pi / 5$, and $N_{1}=N_{2}=3$. Corresponding to these data, we have that $H_{e}=1.282$, $-4 \pi M=0.02556$, and $\max \left(N_{s}\right)=0.9984$. (b) Level curves of $N_{s}$, the density of superconducting charge carriers, for $\kappa=5$, $\bar{B}=2 \pi / 5$, and $N_{1}=N_{2}=3$. Corresponding to these data, we have that $H_{e}=1.327,-4 \pi M=0.07021$, and $\max \left(N_{s}\right)=0.9363$. (c) Level curves of $N_{s}$, the density of superconducting charge carriers, for $\kappa=5, \bar{B}=\pi / 10$, and $N_{1}=N_{2}=3$. Corresponding to these data, we have that $H_{e}=0.4162,-4 \pi M=0.1020$, and $\max \left(N_{s}\right)=0.9990$.

$$
\mathbf{t}_{2}=\left(\frac{4 \pi}{\sqrt{3} \kappa \bar{B}}\right)^{1 / 2}\left(\frac{1}{2} \mathbf{i}_{1}+\frac{\sqrt{3}}{2} \mathbf{i}_{2}\right),
$$

where $i_{1}$ and $i_{2}$ are the unit vectors in the coordinate directions. We make the customary choice of the origin for the point $\mathbf{P}$. The lattice cell $\Omega$ is depicted in Fig. 4 . The only remaining inputs to be chosen are the Ginzburg-Landau parameter $\kappa$, the average magnetic field $\bar{B}$, and the grid information.

The discretized equations are a nonlinear system of algebraic equations. These are solved by a continuation method coupled with Newton's method. The code is configured so that one chooses a fixed value for $\kappa$, and then varies $\bar{B}$. For each pair $(\kappa, \bar{B})$, Newton's method is used to solve the nonlinear equations. The initial guess for Newton's method is determined by continuing from the solution determined for a previous value of $\bar{B}$ and the same value of $\kappa$. The particular continuation method used is a tangent-line approximation to the solution at the previous value of $\bar{B}$. We start with a value of $\bar{B}$ close to the upper limit $\kappa$ for which Newton's method seems to have a large attraction ball; we then continue by successively reducing the value of $\bar{B}$ toward its lower limit 0 .

Computational results were obtained, on a Macintosh II, using piecewise quadratic finite-element functions. A uniform grid spacing was used in each of the $t_{1}$ and $t_{2}$ directions. For each fixed value of $\kappa$, we vary $\bar{B}$. We then obtain approximations for $\zeta$ and $\mathbf{Q}$, which are used to determine $N_{s}$ from (8), $H_{e}$ from (21), and $M$ from (22). The graph of the computed approximation of $-4 \pi M$ vs $H_{e}$ is given in Figs. 5 and 6. In Fig. 5(a), the magnetization for $\kappa=5$, obtained using three intervals in each direction, i.e., $N_{1}=N_{2}=3$, is compared with the corresponding graph for the Monte Carlo-simulated annealing approximation obtained in Doria, Gubernatis, and Rainer. ${ }^{4}$ The agreement is excellent except for low values of the external field. In Fig. 5(b) the magnetization for $\kappa=5$, obtained using three intervals in each direction, is compared with the corresponding graph for four intervals in each direction, i.e., for $N_{1}=N_{2}=4$. The convergence of the finite-element approximations, even for these crude grids, is evident. The same comparisons are repeated in Figs. 6(a) and 6(b) for the case $\kappa=20$.

In Fig. 7 the maximum value of $N_{s}$, the density of superconducting charge carriers, is plotted vs $H_{e} / \kappa$ for three different values of $\kappa$. The graphs for $\kappa=5$ and 20 are virtually indistinguishable. For comparison purposes we also provide the graph for $\kappa=1$. All three plots were obtained with $N_{1}=N_{2}=3$.

The level curves, obtained using $N_{1}=N_{2}=3$, of $N_{s}$, the density of superconducting charge carriers, are given in Fig. 8. The solution in only a single lattice cell was computed; this solution was extended, using (5), to obtain the solution outside the computational cell. The figure captions indicate the values of $\kappa$ and $\bar{B}$ corresponding to each of Figs. 8(a)-8(c), as well as the resulting values of $H_{e}$, $-4 \pi M$, and $\max \left(N_{s}\right)$. The size of each of these three figures is chosen so that they all contain the same number of vortices. Thus the sizes serve as an indication of the spacing between the vortices. Figures $8(a)$ and $8(b)$ corre- 
spond to the same value of $\bar{B}$ but different values of $\kappa$, while Figs. $8(\mathrm{~b})$ and $8(\mathrm{c})$ correspond to the same value of $\kappa$ but different values of $\bar{B}$. A close examination of Figs. $8(\mathrm{a})$ and $8(\mathrm{c})$ reveals that they are virtually scaled versions of each other; note that $\kappa / \bar{B}=50 / \pi$ for both of these figures. Also, note that the ratio of the values of $\kappa$ for these figures is 4 , which, of course, is also the geometric scaling ration between the figures.

\section{ACKNOWLEDGMENT}

The authors wish to thank Dr. James Gubernatis of the Theoretical Division of the Los Alamos National Laboratory for many helpful and interesting discussions, and also for providing the data from Doria, Gubernatis, and Rainer ${ }^{4}$ used in Figs. 5(a) and 6(a).
${ }^{1}$ Q. Du, M. Gunzburger, and J. Peterson, SIAM Rev. 34, 54 (1992).

${ }^{2}$ A. Abrikosov, Zh. Eksp. Teor. Fiz. 32, 1442 (1957) [Sov. Phys. JETP 5, 1174 (1957)].

${ }^{3}$ E. Brandt, Phys. Status Solidi B 51, 345 (1972).

${ }^{4}$ M. Doria, J. Gubernatis, and D. Rainer, Phys. Rev. B 41, 6335 (1990).

${ }^{5}$ G. Eilenberger, Z. Phys. 180, 32 (1964).

${ }^{6}$ W. Kleiner, L. Roth, and S. Autler, Phys. Rev. 133, A1226 (1964).

${ }^{7}$ H. Koppe and J. Willebrand, J. Low Temp. Phys. 2, 499 (1970).

${ }^{8}$ G. Lasher, Phys Rev. A 140, 523 (1965).

${ }^{9}$ F. Odeh, J. Math. Phys. 8, 2351 (1967).

${ }^{10} \mathrm{Q}$. Du, M. Gunzburger, and J. Peterson, SIAM J. Appl.
Math. (to be published).

${ }^{11}$ I. Bubuška and A. Aziz, The Mathematical Foundations of the Finite Element Method with Application to Partial Differential Equations, edited by A. Aziz (Academic, New York, 1972).

${ }^{12}$ P. Ciarlet, The Finite Element Method for Elliptic Problems (North-Holland, Amsterdam, 1978).

${ }^{13} \mathrm{G}$. Strang and G. Fix, An Analysis of the Finite Element Method (Prentice-Hall, Englewood Cliffs, NJ, 1973).

${ }^{14} \mathrm{O}$. Zienkiewicz and R. Taylor, The Finite Element Method (McGraw-Hill, London, 1989).

${ }^{15}$ Q. Du, M. Gunzburger, and J. Peterson, Numer. Math (to be published).

${ }^{16}$ M. Doria, J. Gubernatis, and D. Rainer, Phys. Rev. B 39, 9573 (1989). 